\title{
INFLUÊNCIA DO VOLUME DAS ÁRVORES NO DESEMPENHO DO PROCESSADOR FLORESTAL HARVESTER EM POVOAMENTO DE EUCALIPTO
}

\author{
INFLUENCE OF TREE VOLUME IN THE PERFORMANCE OF HARVESTER FOREST \\ PROCESSOR IN EUCALYPTUS STAND
}

\author{
Carla Krulikowski Rodrigues ${ }^{1}$, Eduardo da Silva Lopes ${ }^{2}$, Diego de Oliveira ${ }^{3}$, Jean Alberto \\ Sampietro ${ }^{4}$
}

1,2,3 Universidade Estadual do Centro-Oeste, Irati, Paraná, Brasil - carlakr@gmail.com, eslopes@unicentro.br\&dioliveira@klabin.com.br

${ }^{4}$ Universidade do Estado de Santa Catarina, Lages, Santa Catarina, Brasil - jean.sampietro@udesc.br

\section{RESUMO}

Objetivou-se avaliar a influência do volume das árvores no desempenho operacional do processador florestal harvester em um sistema de árvores inteiras. O estudo foi realizado em povoamento de Eucalyptus grandis Hill ex. Mainden com 84 meses, submetido ao corte raso e localizado em Telêmaco Borba, Paraná, Brasil. Os dados foram obtidos por meio de estudo de tempos e movimentos para determinar os tempos do ciclo operacional, as disponibilidades mecânica e técnica, a eficiência operacional, a produtividade e os custos de produção. $O$ efeito do volume das árvores na produtividade e nos custos de produção foi avaliado por meio de análise de regressão. Com os resultados obtidos, foi possível verificar que o elemento processamento consumiu $74 \%$ do tempo total efetivo do ciclo operacional. As disponibilidades mecânica e técnica foram de 80,8 e 79,5\%, respectivamente, com uma eficiência operacional de $60,5 \%$. A produtividade média foi de $49,1 \mathrm{~m}^{3}$ he-1, com custo médio de produção de $\mathrm{R} \$ 5,45 \mathrm{~m}^{-3}$. Para a estimativa da produtividade e dos custos de produção, ambos em função do volume das árvores, o polinômio de terceiro grau foi o modelo ajustado, gerando equações com coeficiente de determinação de 0,24 e 0,23, respectivamente. Com isso, mostrou-se que o desempenho operacional da máquina é influenciado diretamente pelo volume das árvores, sendo a produtividade crescente e os custos decrescentes, à medida que há aumento do volume da árvore até aproximadamente $0,6 \mathrm{~m}^{3}$, após este valor, o aumento do volume proporciona efeito inverso no desempenho operacional do harvester.

PALAVRAS-CHAVE: Colheita florestal, Custos, Produtividade.

\begin{abstract}
The aim of this study was to evaluate the influence of tree volume on the operational performance of the harvester processor in an entire tree system. The study was carried out on stand of Eucalyptus grandis Hill ex. Mainden with 84 months, under clear cutting and located in Telêmaco Borba, Paraná, Brazil. The data were obtained by means of a times and motions study to determine the times of the operational cycle, mechanical and technical availability, operational efficiency, productivity and production costs. The effect of tree volume on productivity and production costs was evaluated through regression analysis. With the results obtained, it was possible to verify that the processing element consumed $74 \%$ of the total effective time of the operational cycle. Mechanical and technical availability were 80.8 and $79.5 \%$, respectively, with an operating efficiency of $60.5 \%$. The average productivity was $49.1 \mathrm{~m}^{3} \mathrm{he}^{-1}$, with an average production cost of $\mathrm{R} \$ 5.45 \mathrm{~m}^{-3}$. For the estimation of productivity and production costs, both as a function of tree volume, the third-degree polynomial was the fitted model, generating equations with a coefficient of determination of 0.24 and 0.23 , respectively. With this, it was shown that the operational performance of the machine is influenced directly by the volume of the trees, with increasing productivity and decreasing costs, as there is an increase in tree volume up to approximately $0.6 \mathrm{~m}^{3}$, after this value, the increase in volume provides an inverse effect on the operational performance of the harvester.
\end{abstract}

KEYWORDS: Forest Harvest, Costs, Productivity. 


\section{INTRODUÇÃO}

A colheita da madeira compreende a última etapa do processo produtivo de florestas plantadas, sendo considerada um conjunto de operações realizadas no maciço florestal para a preparação da madeira a ser colocada na indústria (TANAKA, 1986). Tais operações podem ser influenciadas por diversos fatores de ordens técnica, econômica e ambiental (MALINOVSKI et al., 2006; MACHADO et al., 2014).

No Brasil, normalmente as operações de colheita da madeira são realizadas por métodos mecanizados, com o uso de máquinas de elevada capacidade produtiva. Esta característica é encontrada tanto no sistema de toras curtas, quando a madeira é seccionada e depois extraída do interior do povoamento na forma de toras, bem como no sistema de árvores inteiras quando a árvore é cortada e extraída para a margem do talhão para o processamento da madeira.

Dentro do sistema de colheita da madeira de árvores inteiras, o processamento das árvores, ou seja, desgalhamento, traçamento e empilhamento, pode ser feito por diversas máquinas, como slasher, garra traçadora, harvester multifuste ou harvester para árvores individuais (SIMÕES et al., 2014; SCHETTINO et al., 2015). As três primeiras são utilizadas em povoamentos florestais destinados à produção de celulose e energia, quando há pouca exigência na qualidade da mensuração dos produtos gerados. Por outro lado, o harvester para árvores individuais é aplicado onde é requerida elevada qualidade das dimensões das toras, principalmente, em empresas que atuam na geração de multiprodutos (MURPHY, 2013).

O harvester é definido como um trator florestal capaz de derrubar, desgalhar, traçar e empilhar, sendo composto por uma máquina base de pneus ou esteira, com uma grua e um cabeçote (MACHADO et al., 2014). Segundo Robert (2012), no sistema de toras curtas, o harvester realiza a derrubada das árvores e seu processamento dentro do talhão, ao passo que no sistema de árvores inteiras é utilizado apenas para o processamento das árvores dispostas na margem do talhão, sendo caracterizado pela menor necessidade de movimentação.

O harvester também é influenciado diretamente pelo volume da árvore por ciclo operacional, visto que o processamento ocorre individualmente. Muitos trabalhos que envolvem o harvester no sistema de toras curtas na operação de corte foram realizados (SEIXAS \& BATISTA, 2014; LEITE et al., 2014), entretanto, há carência de estudos que avaliam o seu desempenho no sistema de árvores inteiras, em atividade de processamento das árvores.

Além disso, apesar da elevada qualidade das operações de processamento da madeira, o uso do harvester para árvores individuais pode proporcionar baixa produtividade, se comparada as demais máquinas de processamento da madeira, devido as suas características operacionais (DINIZ et al., 2018). Portanto, torna-se necessário conhecer o desempenho operacional do processador florestal harvester para árvores individuais, a fim de auxiliar no planejamento, na seleção das máquinas de colheita de madeira e no dimensionamento da quantidade de máquinas.

Diante disso, objetivou-se neste estudo avaliar o efeito do volume das árvores no desempenho operacional de um harvester na operação de processamento de madeira de eucalipto, contribuindo com informações para o planejamento das operações, aumento de produtividade e redução de custos.

\section{MATERIAL E MÉTODOS}

O estudo foi realizado em áreas operacionais de colheita da madeira em povoamento de Eucalytus grandis Hill ex. Mainden localizado no município de Telêmaco Borba, estado do Paraná, Brasil, entre as coordenadas geográficas $24^{\circ} 19^{\prime} 26^{\prime \prime} \mathrm{S}$ e $50^{\circ} 36^{\prime} 07^{\prime \prime} \mathrm{W}$ e altitude média de $760 \mathrm{~m}$.

O clima do local do estudo, segundo Köppen, é classificado como $\mathrm{Cfa}$, apresentando temperaturas médias inferiores a $18{ }^{\circ} \mathrm{C}$ e superiores a $22^{\circ} \mathrm{C}$, em meses mais frios e quentes, respectivamente. A umidade relativa média anual encontra-se entre 70 a 75\%, com precipitação média anual de 1.400 a $1.600 \mathrm{~mm}$ (ALVARES et al., 2013). O solo predominante nas áreas de estudo é classificado como Latossolo Vermelho-Escuro de textura argilosa, com relevo variando de plano a suavemente ondulado em declividade média de $6 \%$. O regime de corte raso foi adotado no povoamento, sendo a madeira destinada a produção de celulose e papel. As características dendrométricas do povoamento florestal estudado estão descritas na Tabela 1.

O sistema de colheita da madeira utilizado foi o de árvores inteiras, sendo composto por um feller buncher para derrubada das árvores em eitos de quatro linhas, com posterior empilhamento das árvores no interior do talhão. Em seguida, o skidder foi utilizado para o arraste das árvores até a margem do talhão, enquanto o harvester realizou o processamento da madeira no estaleiro em toras de 7,2 $\mathrm{m}$ de comprimento. 
Tabela 1. Características dendrométricas do povoamento.

\begin{tabular}{cc}
\hline Espécie & Eucalyptus grandis \\
\hline Idade de corte (meses) & 84 \\
\hline Espaçamento $(\mathrm{m})$ & $2,5 \times 2,5$ \\
DAP médio $(\mathrm{cm})$ & $19,2 \pm 1,9$ \\
Altura média $(\mathrm{m})$ & $27,6 \pm 2,2$ \\
VMI (m $\left.{ }^{3}\right)$ & $0,366 \pm 0,065$ \\
Volume médio $\left(\mathrm{m}^{3} \mathrm{ha}^{-1}\right)$ & $430,0 \pm 59,4$ \\
\hline
\end{tabular}

Em que: $\mathrm{DAP}$ = diâmetro à 1,3 $\mathrm{m}$ do solo; e $\mathrm{VMI}=$ volume médio individual.

Foi avaliado o processador florestal harvester (Figura 1), composto de máquina base com potência nominal de $219 \mathrm{~kW}$, peso operacional de $31,2 \mathrm{t}$ (sem cabeçote), material rodante de esteiras e vida útil de 10.600 horas. $O$ cabeçote apresentava peso operacional de 2,12t, diâmetro máximo de corte de $750 \mathrm{~mm}$, e com quatro facas de desgalhamento.

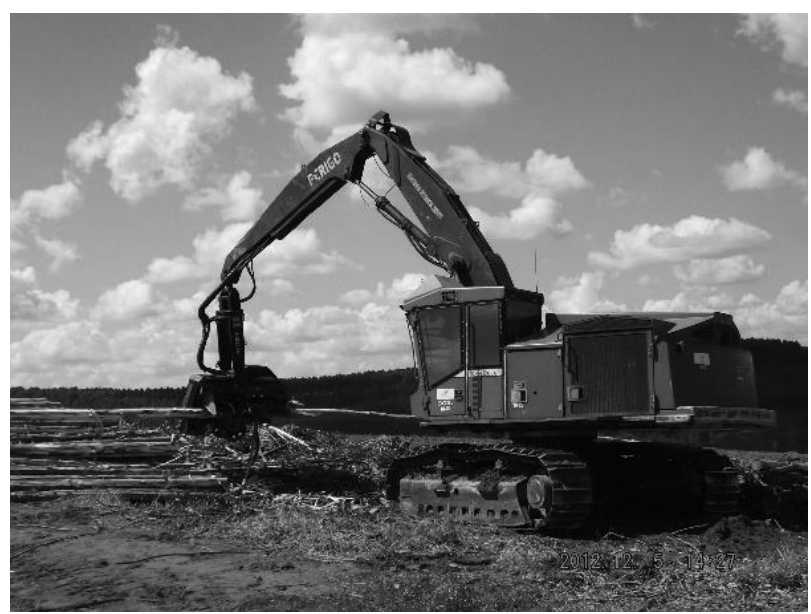

Figura 1. Processador florestal harvester.

A amostragem foi determinada pela metodologia apresentada por Murphy (2005), por meio da realização de um estudo-piloto de tempos e movimentos, obtendo-se o número mínimo de observações do ciclo operacional da máquina, de forma a proporcionar um limite de erro de amostragem máximo de $5 \%$, por meio da equação (1):

$$
\mathrm{n}=\frac{\mathrm{t}^{2} \times C V^{2}}{\mathrm{LE}^{2}}
$$

Em que: $\mathrm{n}=$ número mínimo de ciclos necessários; $\mathrm{t}=$ valor crítico de $t$, para o nível de confiança de $5 \%$; CV = coeficiente de variação (\%); e LE = limite de erro admissível (\%).
O ciclo operacional foi dividido nos seguintes elementos: (1) busca (tempo consumido pela máquina na busca da árvore na pilha de árvores); (2) processamento (tempo consumido pela máquina no processamento das árvores); (3) deslocamento (tempo consumido pela máquina no deslocamento durante o processamento); e (4) interrupções (tempo referente às paradas da máquina por motivos diversos, como manutenção preventiva e corretiva, organização de pilhas, deslocamento, abastecimento, verificação diária e outros).

A disponibilidade mecânica, definida pelo tempo de trabalho em que a máquina esteve mecanicamente apta para realizar o trabalho produtivo, em relação ao tempo programado e desconsiderando os tempos em manutenção preventiva e/ou corretiva, foi obtida pela expressão (2):

$$
\mathrm{DM}=\frac{\mathrm{TP}-\mathrm{TM}}{\mathrm{TP}} \times 100
$$

Em que: $\mathrm{DM}=$ disponibilidade mecânica (\%); $\mathrm{TP}=$ tempo programado para o trabalho (horas); e TM = tempo em manutenção preventiva e corretiva (horas).

A disponibilidade técnica, dada pela relação entre o tempo de trabalho efetivo e o tempo disponível da máquina para o trabalho, foi obtida pela expressão (3):

$$
\mathrm{DT}=\frac{\mathrm{TD}-\mathrm{TI}}{\mathrm{TD}} \times 100
$$

Em que: $\mathrm{DT}=$ disponibilidade técnica (\%); $\mathrm{TD}=$ tempo disponível para o trabalho (horas); e $\mathrm{TI}=$ tempo de interrupções operacionais e não operacionais (horas).

A eficiência operacional expressa a percentagem do tempo de trabalho em que a máquina executou a operação de forma efetiva, desconsiderando os tempos de manutenção preventiva e corretiva e as interrupções operacionais e não operacionais, conforme obtida pela expressão (4):

$$
\mathrm{EO}=\frac{\mathrm{TE}}{\mathrm{TP}} \times 100
$$

Em que: $E O=$ eficiência operacional (\%); TE = tempo de trabalho efetivo (horas); e TP = tempo programado para o trabalho (horas).

A produtividade foi obtida por meio do volume individual das árvores, dividido pelo tempo efetivamente trabalhado, conforme apresentada na expressão (5): 


$$
\operatorname{Pr}=\frac{\mathrm{vi}}{\mathrm{He}}
$$

Em que: $\operatorname{Pr}=$ produtividade $\left(\mathrm{m}^{3} \mathrm{cche} \mathrm{e}^{-1}\right) ;$ vi $=$ volume individual das árvores com casca $\left(\mathrm{m}^{3} \mathrm{cc}\right)$; e He = tempo efetivo de trabalho (horas).

O cálculo dos custos operacionais foi realizado por meio do método contábil, com uso de valores estimados e reais. Os custos fixos (depreciação, juros e seguros) foram estimados pela metodologia proposta por Miyata (1980) e Lopes (2001). Para os custos variáveis (combustíveis, lubrificantes e graxas, óleo hidráulico, pneus, manutenção e reparos) e de pessoal (salário, encargos sociais e transporte) foram obtidos pelo banco de dados da empresa.

O cálculo do custo de produção foi realizado pela divisão dos custos operacionais pela produtividade do processador florestal harvester, conforme apresentada na expressão (6):

$$
\mathrm{CP}=\frac{\mathrm{CO}}{\mathrm{Pr}}
$$

Em que: $C P=$ custo de produção $\left(R \$ \mathrm{~m}^{-3}\right) ; C O=$ custos operacionais $\left(\mathrm{R} \$ \mathrm{he}^{-1}\right)$; e $\operatorname{Pr}=$ produtividade $\left(\mathrm{m}^{3} \mathrm{cc} \mathrm{he} \mathrm{e}^{-1}\right)$.

Realizou-se uma análise estatística descritiva dos valores de produtividade, custos de produção e volume médio das árvores, seguida do ajuste do modelo de polinômio de terceiro grau para expressar a relação entre as variáveis dependentes "produtividade" e "custos de produção" e a independente "volume das árvores". O modelo ajustado foi avaliado por meio do coeficiente de determinação $\left(R^{2}\right)$ e por linhas de tendência apresentadas em gráficos de dispersão.

\section{RESULTADOS E DISCUSSÃO}

O processador florestal harvester foi avaliado por meio de 3.007 ciclos operacionais, sendo necessários apenas 542 para atingir o erro admissível de 5\%. A participação percentual dos tempos produtivos e improdutivos da máquina estão apresentados na Figura 2.

Observou-se que $60 \%$ do tempo programado para a execução do trabalho foi consumido pelos tempos produtivos da máquina. Além disso, dentro do tempo efetivo do ciclo operacional (Figura 2b), 74\% do ciclo foi consumido pela atividade de processamento das árvores, o que corresponde a uma média de 0,37 minutos por árvore.

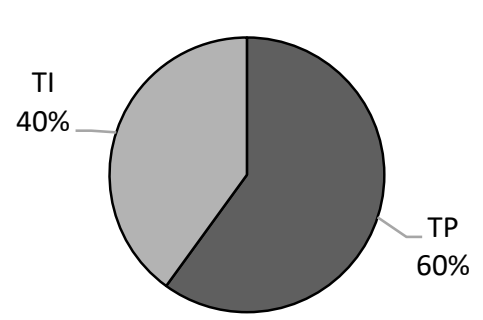

(a)

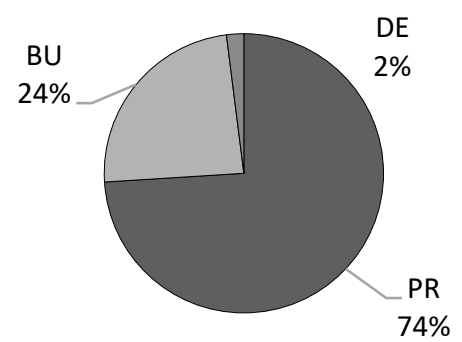

(b)

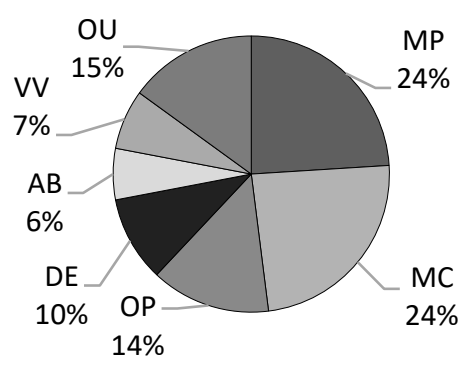

(c)

Em que: $\mathrm{TP}=$ tempos produtivos; $\mathrm{TI}=$ tempos improdutivos; $\mathrm{BU}=$ busca; $\mathrm{DE}=$ deslocamento; $\mathrm{PR}=$ processamento; $\mathrm{MP}=$ manutenção preventiva; $\mathrm{MC}=$ manutenção corretiva; $\mathrm{OP}=$ organização de pilhas; $\mathrm{DE}=$ deslocamento; $\mathrm{AB}=$ abastecimento; $\mathrm{VV}=$ verificação diária; e $\mathrm{OU}=$ outros.

Figura 2. Participação percentual de tempos produtivos e improdutivos do processador florestal harvester (a); participação percentual dos elementos de ciclo operacional efetivo (b); e participação percentual das interrupções operacionais ocorridas nos tempos improdutivos (c).

O tempo de busca de árvore nas pilhas de madeira, representou $25 \%$ do tempo total do ciclo, o que correspondeu a 0,12 minutos. Por outro lado, os deslocamentos da máquina consumiram apenas $2 \%$ do tempo total, sendo um resultado esperado devido às características operacionais da atividade, onde geralmente a máquina realiza o trabalho com poucos movimentos na margem do talhão.

Nos tempos improdutivos, as interrupções (Figura 2c) foram causadas, principalmente, pelas manutenções da máquina (48\%), onde a maior parte do tempo consumido pela manutenção mecânica foi causada pela necessidade de trocas de correntes e sabres, seguido de trocas das mangueiras do sistema hidráulico do implemento.

As disponibilidades mecânica e técnica obtidas foram de 80,8 e $79,5 \%$, respectivamente, o que resultaram em 
eficiência operacional de aproximadamente $60,5 \%$. Já a produtividade média do processador florestal harvester por hora efetiva foi de $49,1 \mathrm{~m}^{3} \mathrm{cc}$, ao passo que o custo de produção médio foi de $\mathrm{R} \$ 5,45$ por $\mathrm{m}^{3}$, conforme apresentado na Tabela 2.

Tabela 2. Estatística descritiva das variáveis dependentes e indenpendente para estimativa da produtividade e custos de produção do processamento florestal harvester.

\begin{tabular}{cccccc}
\hline Variável & Min. & Med. & Média & Máx. & DP \\
\hline $\operatorname{Pr}\left(\mathrm{m}^{3} \mathrm{cc} h \mathrm{~h}^{-1}\right)$ & 11,7 & 47,5 & 49,1 & 100,2 & 16,8 \\
$\mathrm{CP}\left(\mathrm{R} \$ \mathrm{~m}^{-3}\right)$ & 2,35 & 4,95 & 5,45 & 20,04 & 2,20 \\
$\operatorname{vi}\left(\mathrm{m}^{3}\right)$ & 0,290 & 0,350 & 0,366 & 0,680 & 0,065 \\
\hline
\end{tabular}

Em que: $\mathrm{Pr}=$ produtividade efetiva; $\mathrm{CP}$ = custo de produção; vi = volume individual das árvores; $\mathrm{DP}=$ desvio padrão; Med. = mediana; Máx. = máximo; e Min. = mínimo.

A produtividade média obtida foi superior aos valores obtidos por Seixas \& Batista (2014), que ao avaliarem diferentes modelos de harvester com rodados de pneus e esteiras na operação de corte em sistema de toras curtas em povoamento de Eucalyptus sp., encontraram produtividade de 12,7 a $36,1 \mathrm{~m}^{3} \mathrm{~h}^{-1}$. Leite et al. (2014), ao estudarem o desempenho do harvester na operação de corte de Eucalyptus sp. em diferentes espaçamentos e declividades, observaram produtividade de 25,6 a $31,5 \mathrm{~m}^{3} \mathrm{he}^{-1}$.

Nota-se que a produtividade e os custos de produção da máquina foram influenciados diretamente pelo volume individual das árvores (Figura 3).

Com o modelo de polinômio de terceiro grau ajustado, tanto para a estimativa da produtividade quanto para os custos de produção, verificou-se acréscimo do desempenho operacional da máquina com o aumento do volume das árvores, resultando, consequentemente, em redução dos custos de produção. Entretanto, é possível observar este comportamento em um volume médio da árvore de aproximadamente $0,6 \mathrm{~m}^{3}$, em que, após este valor, ocorreu um comportamento inverso.

Segundo Leite et al. (2013), a capacidade produtiva do harvester se eleva proporcionalmente com o aumento do volume das árvores, acarretando em uma tendência inversa para os custos de produção, Burla et al. (2012) comprovaram que a produtividade se eleva com o incremento do volume individual das árvores até um ponto máximo, decrescendo posteriormente a este valor. Isto se deve ao fato de que as árvores apresentam um volume elevado, o que dificulta o trabalho da máquina, demonstrando o seu limite operacional.
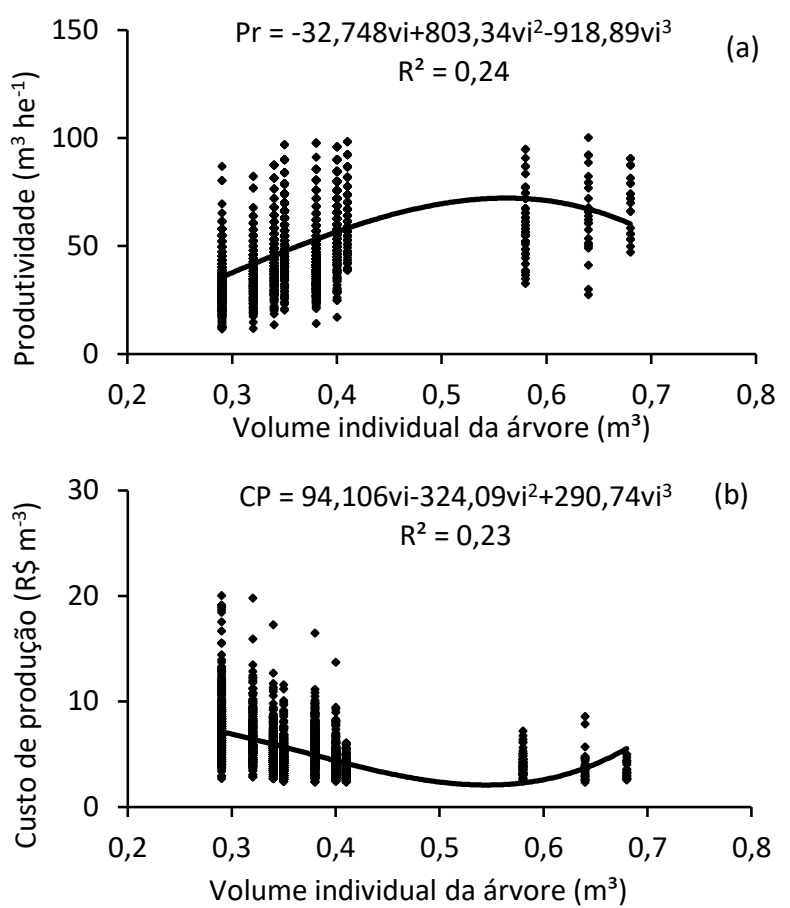

Em que: $\operatorname{Pr}=$ produtividade $\left(\mathrm{m}^{3} \mathrm{cc}\right.$ he $\left.\mathrm{e}^{-1}\right) ; \mathrm{vi}=$ volume individual das árvores com casca $\left(\mathrm{m}^{3} \mathrm{cc}\right)$; e $\mathrm{CP}=$ custo de produção $\left(\mathrm{R} \$ \mathrm{~m}^{-3}\right)$.

Figura 3. Distribuição dos valores de produtividade efetiva (a) e custos de produção (b) em função do volume individual da árvore.

\section{CONCLUSÕES}

O ciclo operacional efetivo do processador harvester foi ocupado em grande parte do tempo pelo processamento efetivo da árvore, demonstrando que as características das árvores afetam o desempenho da máquina na execução da operação; e

A produtividade da máquina e os custos de produção foram diretamente afetados pelo volume da árvore até o limite de $0,60 \mathrm{~m}^{3}$, demostrando a importância desta variável ser considerada no planejamento operacional.

\section{REFERÊNCIAS}

ALVARES, C. A. et al. Köppen's climate classification map for Brazil. Meteorologische Zeitschrift, v. 22, n. 6, p. 711-728, 2013.

BURLA, E. R. et al. Avaliação técnica e econômica do harvester em diferentes condições operacionais. Engenharia na Agricultura, $v$. 20, n. 5, p. 412-422, 2012.

DINIZ, C. C. C. et al. Avaliação técnica de cabeçotes individual e múltiplo no processamento de madeira. Advances in Forestry Science, v. 5, n. 1, p. 253-258, 2018.

LEITE, E. S. et al. Desempenho do harvester na colheita de eucalipto em diferentes espaçamentos e declividades. Árvore, v. 38, n. 1, p. 1-7, 2014.

LEITE, E. S. et al. Modelagem técnica e de custos do Harvester no 
corte de madeira de eucalipto no sistema de toras curtas. Scientia Forestalis, v. 41, n. 98, p. 205-215, 2013.

LOPES, E. S. Aplicação do programa SNAP III (Scheduling and Network Analysis Program) no planejamento da colheita e do transporte florestal. 2001. 150 p. (Tese de doutorado)

MACHADO, C. C.; SILVA, E. N.; PEREIRA, R. S.; CASTRO, G. P. O setor florestal brasileiro e a colheita florestal. Colheita florestal. 3 ed. Viçosa: UFV, 2014.

MALINOVSKI, R. A. et al. Análise das variáveis de influência na produtividade das máquinas de colheita de madeira em função das características físicas do terreno, do povoamento e do planejamento operacional florestal. Floresta, v. 36, n. 2, p. 169182, 2006.

MIYATA, E. S. Determining fixed and operating costs of logging equipament. Washington: USDA Forest Service, 1980.

MURPHY, G. Determining sample size for harvesting cost estimation. New Zealand Journal of Forestry Science, v. 35, n. 1/2, p. 166-169, 2005.

MURPHY, G. Procedures for scanning radiata pine stem dimensions and quality on mechanised processors. International Journal of Forest Engineering, v. 14, n. 2, p. 91-101. 2013.

ROBERT, R. C. G. Guia prático de operações florestais na colheita de madeira. Curitiba: Ed. do Autor, 2012.

SCHETTINO, S. et al. Correlação entre volumetria de florestas de eucalipto e produtividade e custos de máquinas de colheita de madeira. Árvore, v. 39, n. 5, p. 935-942, 2015.

SEIXAS, F.; BATISTA, J. L. F. Comparação técnica e econômica entre harvesters de pneus e com máquina base de esteiras. Ciência Florestal, v. 24, n. 1, p. 185-191, 2014.

SIMÕES, D. et al. Produtividade e custos do feller-buncher e processador florestal em povoamento de eucalipto de primeiro corte. Ciência Florestal, v. 24, n. 3, p. 621-630, 2014.

TANAKA, O. P. Exploração e transporte da cultura do eucalipto. Informe Agropecuário, n. 141, p. 24-30, 1986. 